\title{
The development and trend of wireless body area networks in the smart clothing field
}

\author{
Tan Dou ${ }^{1, a}$ Liu Yi ${ }^{1, b}$ \\ ${ }^{1}$ Beijing Institute of Fashion Technology, Chaoyang District, Beijing, China

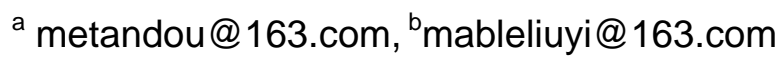

Keywords:Wireless body area network. Smart clothing. IEEE 802.15.4. IEEE 802.15.6

Abstract. With the advent of the smart clothing, wireless body area network(WBAN) has became particularly important as the information perception stage of smart clothing. Firstly, we introduced the conception of the smart clothing and the wireless body area network briefly. And then we analyzed many traits about two protocols -IEEE 802.15.4 and IEEE 802.15.6-which can be used in the WBAN.Finally, we introduced the application of WBAN in the area of smart clothing. The combination of WBAN with smart clothing will play a very important role in the future human life.

\section{Introduction}

With the rapidly development of information era, not only beautiful, fashion, quality is the people required in clothing, but also self-control.Therefor the smart clothing is appeared. As the carrier supporter of the sensors, smart clothing can collect human body data and relevant environment data where we stay. As the information perception and the transmission stages of smart clothing, sensor network especially wireless body area network(WBAN) is extraordinarily significant.

\section{Smart clothing}

Smart clothing is a life simulation systems, a clothing with the ability of perception and response[1]. The sensors in or on the clothing can collected data, then disposed it. Smart clothing is a advanced technology which combined many other technologies about sensor, electronic,information, material, and textile and so on. In sum, it can be divided two domains, one is changed the structure of textile materials by physical and chemical methods, it can make the textile materials having some new functions[2]. Another is combined smart clothing with information technology, it can brought some functions to clothing, which can satisfied people' s requirement[3].

Smart clothing is began with a fund project of united states navy, named Georgia Tech Wearable Motherboard(GTWM), in 1996[4].The first generation clothing is used ordinary drapery and electron components. They put electron components on the drapery immediately, it is very hard to disassemble and wash. Hence the researchers make some improvement, they lessen the size of electron components by micro-electronics technology. The second generation clothing is the integration of intelligent components and fibers, we can put the components in the fibers according what functions we needed.Smart clothing will become more functionalization. Numerous experts argue that the study of smart clothing will make a huge difference in textile industry and semiconductor area, even our lifestyle,in the future. This clothing is the trend of future textile and garment domain[5]. 


\section{WBAN}

WBAN,also called Body Area Sensor Networks(BASN)[6], is a communication network which is consist of many network elements, for instance, personal terminal, network equipment, and the sensors which is distributed on body or interior or the surroundings[7]. The information and data which is collected by the sensors is transmitted to terminal and disposed it, than the processed data is communicated with the external network.

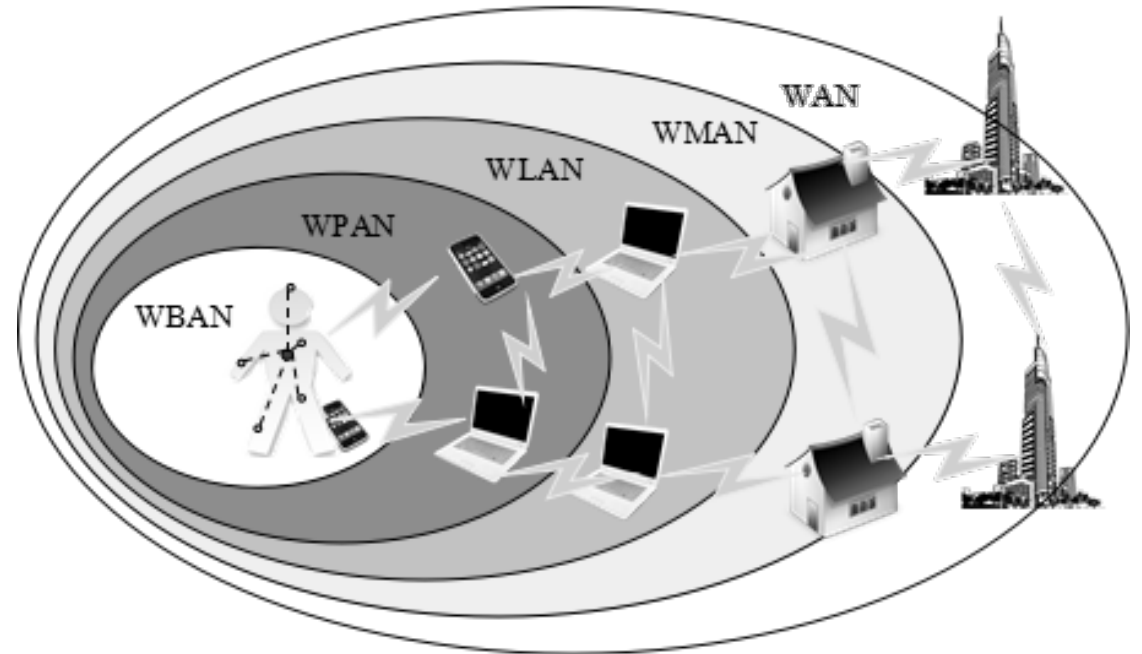

WPAN: wireless personal area network WLAN: wireless local area network

WMAN: wireless metropolitan area network WAN: wide area network

Fig. 1 The relationship of WBAN and other wireless network technologies

The relationship of WBAN, WPAN, WLAN, WMAN, WAN is shown in Figure 1[8]. WBAN is the communication technology of body surface or interior, it has the shortest communication distance, just 2 to 3 meters.WPAN is about the human's carry-on electronic equipment like telephone, computer, under 10 meter. The communication distance of WLAN can usually reach hundred meters. WMAN is used to solve the problem about the access of metropolitan area network. Its distance can reach several thousand meters. WAN is the longest,it supports data exchange from a lot of cities, even many states.

\section{Development}

\section{Standards}

WBAN has many unique advantages,compered to the traditional wireless sensor network[9]. Its equipment is very small, it is impossible to change the battery. So the energy consumption and storage capacity of equipment is needed. It is so close between every component that it' s easy to generate interference each other, therefore, it' $s$ necessary for this equipment to have low-power transmission, and than reduce interference. If we put WBAN to medical treatment, the system should be a real-time and high reliability system. In a word,the technologies applied to WBAN should have low delay, high reliability, low power consumption and so on.According this demands, once the IEEE 802.15 working group has set two standards for WBAN, IEEE 802.15.4 and IEEE 802.15.6.

IEEE 802.15.4 came from TG4, the task group in the IEEE802.15 working group, and published in October, 2003.It's a standard for the low-rate wireless personal area network(LR-WPAN). It was aimed at providing a common standard for the low-late interconnection of persons or families. IEEE 802.15.6 is the first global WBAN standard, there were about sixty manufacturer engineers made 
efforts to set this standard. It could provide a low-power consumption,high reliability, inexpensive, uncomplicated, communication technology in human body, surface, and external[10]. The comparison of two protocols is shown in Table 1.

Table 1 IEEE802.15.4 and IEEE 802.15.6

\begin{tabular}{|c|c|c|}
\hline & IEEE802.15.4 & IEEE802.15.6 \\
\hline $\begin{array}{l}\text { Network } \\
\text { topology }\end{array}$ & Star,point-to-point & Star(one-hop,two-hop) \\
\hline Physical layer & $\begin{array}{c}\text { Two kinds: } 2.45 \mathrm{GHz} \text { and } \\
868 / 915 \mathrm{MHz}\end{array}$ & $\begin{array}{c}\text { Three specification: } \\
\text { Narrowband(NB), Ultra } \\
\text { wideband(UW), Human body } \\
\text { communications(HBC) }\end{array}$ \\
\hline $\begin{array}{l}\text { Frequency } \\
\text { bond }\end{array}$ & $\begin{array}{l}\text { Sixteen } 2450 \mathrm{MHz} \text {, one } \\
915 \mathrm{MHz} \text {, one } 868 \mathrm{MHz}\end{array}$ & $\begin{array}{c}\text { Embedded equipment: } \\
\text { 402-405MHz } \\
\text { Body surface: } 2.4 \mathrm{GHz} \text {, } \\
\text { 3.1-10.6GHz }\end{array}$ \\
\hline Access method & Support CSMA/CA & CSMA/CA,slot Aloha,eta \\
\hline Object & LR-WPAN & WBAN \\
\hline $\begin{array}{c}\text { MAC frame } \\
\text { format }\end{array}$ & Figure 2 & Figure 3 \\
\hline Modulation & $\begin{array}{c}\text { 2.45GHz PHY layer: } \\
\text { O-QPSK, } \\
\text { 868/915MHz PHY layer: } \\
\text { BPSK }\end{array}$ & $\begin{array}{c}\text { NB PHY layer:DBPSK, GMSK, } \\
\text { DQPSK, D8PSK, } \\
\text { UWB PHY layer: } \\
\text { on-off signaling,CP-BFSK, } \\
\text { wideband FM,DPSK } \\
\end{array}$ \\
\hline
\end{tabular}

Both star and point-to-point network topology could be used to the IEEE 802.15.4, but IEEE 802.15.6 just support the star topology with one-hop or two-hop. Because the centralized management of WBAN is needed, so the topology of WBAN should be star. Both of two standards were all suit WBAN.IEEE 802.15.4 was chose the Industrial Scientific Medical frequency(ISM) bond,this bond was oriented $\mathrm{f}$ or this three organizations,industrial, scientific, and medical. But, the more wireless communication technologies chose this bond, the more chance of interference.

Fig. 2 The MAC frame format of IEEE 802.15.4

Octet:

Variable-length 2

\begin{tabular}{|c|c|c|c|c|c|c|c|}
\hline \multicolumn{6}{|c|}{ MAC Header } & \multirow{3}{*}{$\begin{array}{l}\text { MAC } \\
\text { Frame } \\
\text { Body }\end{array}$} & \multirow{3}{*}{ FCS } \\
\hline \multirow{2}{*}{$\begin{array}{l}\text { Frame } \\
\text { Control }\end{array}$} & \multirow{2}{*}{$\begin{array}{c}\text { Frame } \\
\text { Sequence } \\
\text { Number }\end{array}$} & $\begin{array}{c}\text { Target } \\
\text { PAN ID }\end{array}$ & $\begin{array}{c}\text { Target } \\
\text { Address }\end{array}$ & $\begin{array}{c}\text { Source } \\
\text { ID }\end{array}$ & $\begin{array}{c}\text { Source } \\
\text { Address }\end{array}$ & & \\
\hline & & \multicolumn{4}{|c|}{ Address } & & \\
\hline
\end{tabular}


Fig. 3 The MAC frame format of IEEE802.15.6

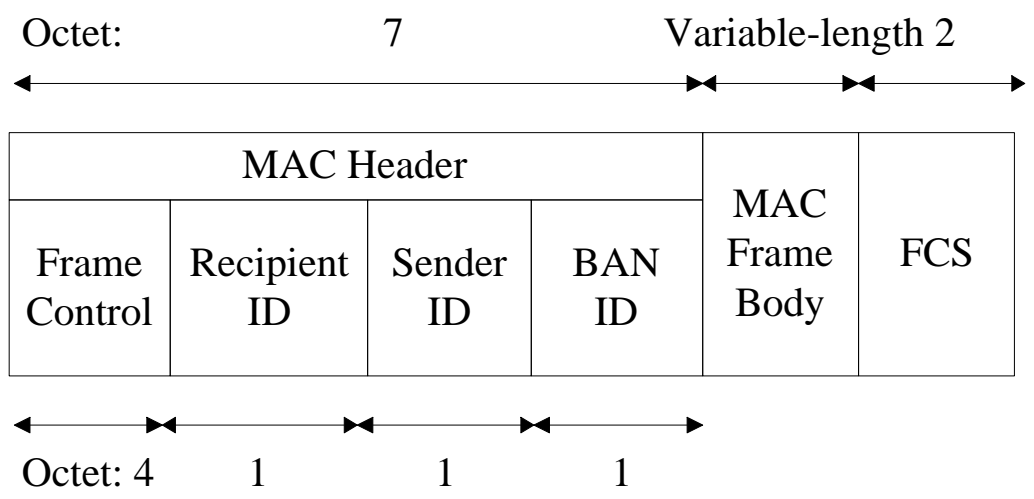

CSMA/CA could be used to IEEE 802.15.4, although IEEE 802.15.4 was also can achieve the goals of WBAN, like low-power consumption, high reliability, and low delay, there was some shortages in it. One is the stationary, limited slot couldn' t access numerous nodes. Anther is the reliability of data transmission was still have some problem in the MAC layer.Hence references [11] was put forward the mini-slot ALOHA to solve this problems.

The MAC frame format of this two protocols were shown in Figure 2 and Figure 3, it is easy to found two MAC frame format were similar. They were all constituted with three parts, MAC header, MAC frame body, and Frame Check Sequence(FCS), but there still were many difference with this two protocols. WBAN is used to human body, the data we transmitted was the personal physiological information like pulse, blood pressure, it is particularly private,just for someone. Therefore a high safe security mechanism was needed for WBAN. There were three security levels be defined in the MAC frame control from the MAC header in standard IEEE 802.15.16. Level 0 was unsecured communication, level 1 was authentication but not encryption, level 2 was authentication and encryption[10].

\section{Applications}

With the development of the WBAN technology, it is applied more and more widely in the field of smart clothing, and it can be roughly divided into three aspects.

i. Aid to disabled people.

If we use the WBAN in the disabled people, it could lead a good effect on their disadvantages. For example, the researchers has designed a smart warm trousers in references[12]. They embed the temperature sensor and the heating wire into clothing material, then used the external temperature control system to control the heating and keeping warm. This trousers can help disabled people defense cold for the muscle atrophy and poor blood circulation.

ii.Health.

Human health has been the primary problem which people concern, and WBAN in the field of health could diagnose person's physical condition, and brought a large of convenience to human beings. While, researchers in references[13], they put forward a kind of mood distinguishing model for smart clothing, they also used sensors in the clothing, to collect human physiological information, such as electrocardio(ECG), breathe, temperature, etc. They distinguished the person's mood by taking feature extraction and processing information. And the scholars in references[14] designed a kind of sub-health intelligent evaluation system based on ECG monitoring of smart clothing. They evaluated and classified the sample by using support vector machine (SVM). This system has played a role in sub-health treatment.

iii.Medical. 
The sensors on smart clothing could be used to collect all kinds of human physiological data. The processed data will be sent to the hospital or patients and patients' families by WBAN and external network. Not only could the hospital understand and track the situation of the patient every time, enhance the working efficiency of the hospital, but also could let the patients or their family members understand the patient's health, particularly deal with emergencies in time, such as, the patient falling down suddenly, heart attack, etc. Figure 4 was shown the architecture of WBAN, it was useful to explain the smart clothing and WBAN in medical application better.

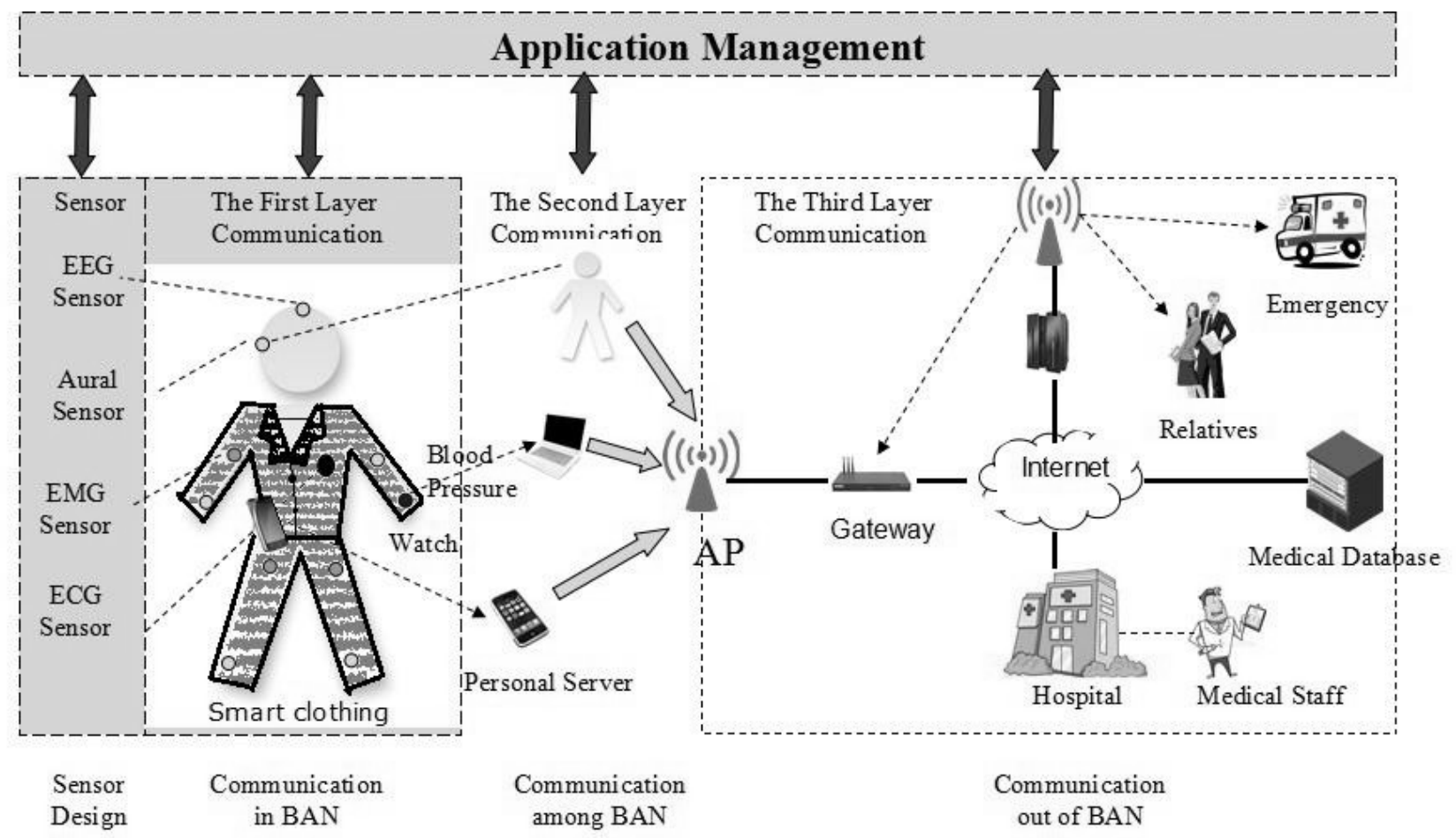

Fig.4 The architecture of WBAN

\section{Summary}

International Standard Organization(ISO) made the IEEE 802.15.6 standard for WBAN, this standard were all suited the demand of WBAN. But it still couldn't play a perfect role in WBAN, especially it was published at 2012. On the contrary, IEEE 802.15.4 was exist 12 years, a lot of technologies have been used to WBAN,like Zigbee. Now the world is becoming diversification,the communication is also too. We should combine with many other technologies to increase the quality of WBAN rather than a single technology. Similarly the application of WBAN in the field of smart clothing will also become wider and wider with the development of other relevant technologies. It will also bring us a better life style.

\section{References}

[1] Tian Miao, Li Jun. Design mode and development tendency of smart clothing[J].Journal of Textile Research, 2014,35(2):109-115.

[2] Mather R R.The exploitation and application of smart textile[J]. 2002, 11:1-3.

[3] Murthy H V S.The introduction of smart textile[J].Textile technology overseas,2003,12:1-5.

[4] Park S,Gopalsamy C,Rajamanickam R,et al. The wearable motherboard: an information infrastructure or sensate liner for medical application[J].Studies in Health Technology and Informatics, 1999,62:252-258. 
[5] Zhai Xiu Mei,Xu Jun.The development and future of smart clothing[J].New Textile, 2003 (5): 14-16.

[6] Ehyaie A,Hashemi M,Khadivi P.Using Relay Network to Increase Life time in Wireless Body Area Sensor Networks[C]Kos:Proceedings of International Symposium on a World of Wireless,Mobile and Multimedia Networks,2009:1-6.

[7] KARAOGUZ J. Hish rate wireless personal area networks[J]. IEEE Comm.Magazine, 2001,9(12):96-1102.

[8] Lu Kai.The research of key technology to wireless body area network(WBAN)[D]. Nanjing: Nanjing University of Posts and Telecommunications,2009.

[9] Milenkovi ć A, Otto C, Jovanov E. Wireless sensor networks for personal health monitoring: Issues and an implementation[J]. Computer communications, 2006, 29(13): 2521-2533.

[10]802.15.6-2012 - IEEE Standard for Local and metropolitan area networks -Part 15.6: Wireless Body Area Networks. 29 February 2012. pages: 1-271.

[11] Wang Lingling.Study of Access Protocols in Wireless Body Area Networks based on IEEE 802.15.4[D]. Xi’an: Xidian University, 2011.

[12] Yang Weixing. Design And Research of Intelligent Warm Pant Temperature Control System Based On Wireless Data Communication[D]. Beijing Institute of Fashion Technology, 2012.

[13]Wu Xuekui, Ren Lihong, Ding Yongsheng et al. Multi-physiology information fusion for emotion distinction in smart clothing[J]. Computer Engineering and Applications, 2009, 45(33): 218-221.

[14] Shi Jinlan, Ren Lihong, Ding Yongsheng et al. Sub-health intelligent evaluation system based on ECG monitoring of smart clothing[J]. Microcomputer Applications. 2008, 24(1): 1-3.

[15]Ding Yongsheng, Wu Yizhi, Hao Kuangrong,Ren Lihong et al. The theory and application of smart clothing[M].Beijing:Science press,2013. 